\title{
Color mixing models for smart lighting systems based on RGBW and WW LEDs
}

\author{
V.I. Kornaga ${ }^{1}$, V.M. Sorokin ${ }^{1}$, A.V. Rybalochka ${ }^{1}$, O.S. Oliinyk ${ }^{1}$, N.P. Kornaga ${ }^{2}$ \\ ${ }^{I}$ V. Lashkaryov Institute of Semiconductor Physics, National Academy of Science of Ukraine, \\ 41, prospect Nauky, 03028 Kyiv, Ukraine \\ ${ }^{2}$ National Technical University of Ukraine "KPI”, Kyiv, Ukraine \\ E-mail:kornaga@isp.kiev.ua
}

\begin{abstract}
Color mixing models to obtain white light with a predetermined values of correlated color temperature and brightness are presented. One model describes mathematical algorithm for combination of spectra for WW LEDs lighting systems, and other one for systems based on RGBW LEDs. Results of testing of created sample of LED light source for indoor lighting ("Amstrong” type) with implemention of presented color mixing models are shown.
\end{abstract}

Keywords: smart lighting system, color mixing, RGBW and WW LEDs, correlated color temperature, brightness, Planckian locus.

Manuscript received 12.03.15; revised version received 18.06.15; accepted for publication 03.09.15; published online 30.09.15.

\section{Introduction}

To date, the most promising in terms of providing energy efficiency and lighting quality are the so-called smart lighting systems (SLS). These systems are not just lighting devices, but programmable complex, the main point of which is the automated control of the lighting parameters and, if necessary, cooperation with other similar systems that are included, for example, into the complex "smart house". SLS typically have opportunities to operate in automatic mode, manual adjustments and settings modes, to change the brightness and color parameters according to a given algorithm. Also, using them one can implement algorithms to change the lighting characteristics according to the natural cycle, which are based on the data of recent studies on the impact of dynamical changes in the spectral parameters of natural lighting on the concentration of the hormone melatonin in the human blood, which is a regulator of the states of cheerfulness and human sleep $[1,2]$.

Among the large number of existing today light sources for creating SLS, there are most suitable LEDs that are both solid and based on organic light-emitting diodes (OLED) [3]. OLED prevail through the absence of spot light and possibility of creating light surfaces with almost arbitrary shape and size, but their efficiency is still low, and the cost is high. Solid-state LEDs, unlike the structures OLED, have high luminous efficiency and moderate price, and that is why they are the best solution to date for applying in SLS, even if the use of the auxiliary optics and structural elements needed to create the desired lighting effects leads to some reduction in the energy efficiency of the system as a whole.

To implement the changes in the spectrum and intensity of radiation by using SLS in accordance with the natural dynamics of the change in spectral composition of daylight, in the most simple case it is sufficient to use two types of white luminophore LEDs with two different values of correlated color temperature (WW system) [4]. The use for this purpose RGB LEDs [5] provides to obtain except controlled white light also a large range of colors, which enables in these systems to implement additional features such as decorative lighting and signaling. A significant drawback of the systems built on RGB LEDs is a low value of the color rendering index $R_{a}(\mathrm{CRI})$ when 
obtaining white light. A variant, in which one can get high-quality white light in SLS with the possibility to reproduce a large range of other colors, is their construction on RGBW and RGBA LEDs [6].

This paper shows the ways of obtaining white light with predetermined values of correlated color temperature and chromaticity coordinates that are maximal close to the Planckian locus based on modeling spectral characteristics when using RGB LEDs in combination with different types of white (WW) LEDs. An example of practical implementation of mathematical models created by mixing colors on the test samples WW RGBW and smart lighting systems.

\section{Mathematical model for combination of spectra for two white luminophore LEDs}

When mixing emission of two light sources (e.g., LED1, $x_{L E D 1}, y_{L E D 1}$ and LED2 $x_{L E D 2}, y_{L E D 2}$ ), one can obtain light with a range of possible colors, which, in the diagrams of color space CIE 1931 and CIE 1960, falls into direct segment LED1-LED2 between the chromaticity coordinates of these two light sources (Fig. 1) [7]. That is why when mixing light of two WW LEDs, one can get no more than two variants of all possible ones of white colors, the chromaticity coordinates of which correspond directly to the Planckian locus values. For all other colors, one can speak only about determining the certain value of correlated color temperature for them.

To obtain light of a correlated color temperature, it is necessary to find a point on the segment LED1-LED2, in which this point corresponds to the desired color temperature in the Planckian locus. For this purpose, we must go to the chromaticity diagram CIE 1960 and to find the coordinates of the desired color temperature by the following formulas:

$u=\frac{4 x}{-2 \tilde{o}+12 \dot{o}+3}, v=\frac{6 \dot{o}}{-2 \tilde{o}+12 \dot{o}+3}$.

The cross-section of perpendicular to the Planckian locus in the point with a certain color temperature and the segment LED1-LED2 determines a point with the corresponding correlated color temperature. Knowing the chromaticity coordinates of LEDs, the equation of this straight line can be found as follows:

$\frac{u-u_{L E D 1}}{u_{L E D 2}-u_{L E D 1}}=\frac{v-v_{L E D 1}}{v_{L E D 2}-v_{L E D 1}}$,

where $u$ and $v$ are the coordinates of chromaticity diagram CIE 1960, $x_{L E D 1}, y_{L E D 1}$ and $x_{L E D 2}, y_{L E D 2}$ are the chromaticity coordinates of the first and second LED, respectively.



Fig. 1. The segment with the possible colors when mixing light of two white LEDs in the color diagram CIE 1931.

The equation of perpendicular to the Planckian locus at the point with the coordinates $\left(u_{0}, v_{0}\right)$ takes the following form:

$v-v_{0}=-\frac{1}{f^{\prime}\left(u_{0}\right)}\left(u-u_{0}\right)$,

where $f^{\prime}\left(u_{0}\right)$ is the derivative of the function that approximates the Planckian locus.

Since the implementation of this function in hardware requires some computing resources, and for practical implementation in SLS, it is important to minimize them, then simplification of the calculation of perpendicular to the Planckian locus at the point $\left(u_{0}, v_{0}\right)$ is possible through determining it as perpendicular to the straight line that goes through two adjacent points located in the Planckian locus from both sides to this point with known chromaticity coordinates $\left(u_{1}, v_{1}\right)$ and $\left(u_{2}, v_{2}\right)$ (Fig. 2):

$v=-u \frac{\left(u_{2}-u_{1}\right)}{\left(v_{2}-v_{1}\right)}+u_{0} \frac{\left(u_{2}-u_{1}\right)}{\left(v_{2}-v_{1}\right)}+v_{0}$.

With the equations for perpendicular passing through the point $\left(u_{0}, v_{0}\right)$ and the straight line passing through the coordinates of two luminophore LEDs $\left(u_{L E D 1}, v_{L E D 1}\right)$, $\left(u_{L E D 2}, v_{L E D 2}\right)$, one can find the coordinates of their intersection point with the corresponding value of correlated color temperature to the value at the point $\left(u_{0}, v_{0}\right)$ :

$$
\begin{aligned}
& v_{c}=\frac{\left(v_{0}-u_{L E D 1}\right) \cdot\left(v_{L E D 2}-v_{L E D 1}\right) \cdot\left(u_{2}-u_{1}\right)+\left(v_{2}-v_{1}\right) \cdot\left(v_{L E D 2}-v_{L E D 1}\right) \cdot v_{0}+v_{L E D 1} \cdot\left(u_{L E D 2}-u_{L E D 1}\right) \cdot\left(u_{2}-u_{1}\right)}{\left(u_{L E D 2}-u_{L E D 1}\right) \cdot\left(u_{2}-u_{1}\right)+\left(v_{2}-v_{1}\right) \cdot\left(v_{L E D 2}-v_{L E D 1}\right)} \\
& u_{c}=\frac{\left(u_{2}-u_{1}\right) \cdot\left(u_{L E D 2}-u_{L E D 1}\right) \cdot u_{0}+\left(v_{0}-v_{L E D 1}\right) \cdot\left(v_{2}-v_{1}\right) \cdot\left(u_{L E D 2}-u_{L E D 1}\right)+u_{L E D 1} \cdot\left(v_{L E D 2}-v_{L E D 1}\right) \cdot\left(v_{2}-v_{1}\right)}{\left(v_{L E D 2}-v_{L E D 1}\right) \cdot\left(v_{2}-v_{1}\right)+\left(u_{2}-u_{1}\right) \cdot\left(u_{L E D 2}-u_{L E D 1}\right)}
\end{aligned}
$$




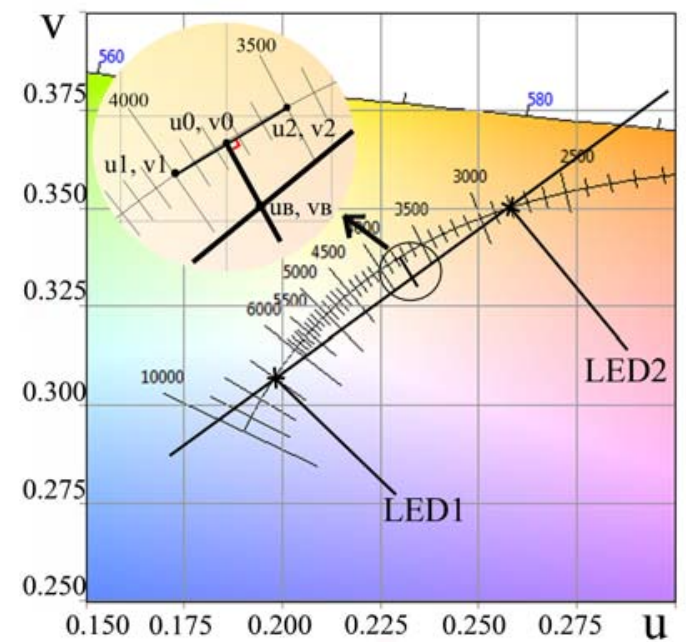

Fig. 2. Method for obtaining the coordinates of points with the given correlated color temperature in the interval LED1-LED2.

These coordinates are now required to recalculate into the coordinates of the chromaticity diagram CIE 1931:

$x_{c}=\frac{3 u_{c}}{2 u_{c}-8 v_{c}+4}, y_{c}=\frac{2 v_{c}}{2 u_{c}-8 v_{c}+4}$.

It is possible to find the corresponding coordinates $X_{c}, Y_{c}, Z_{c}$, taking that the coordinate $Y_{c}$ (brightness) is equal to unity, that is:

$Y_{c}=1, \quad X_{c}=\tilde{o}_{c} \cdot \frac{Y_{c}}{o_{c}}, Z_{c}=\left(1-x_{c}-y_{c}\right) \frac{Y_{c}}{y_{c}}$.

In the same way, one can find the coordinates of two luminophore LEDs $X_{L E D 1}, Y_{L E D 1}, Z_{L E D 1}, X_{L E D 2}, Y_{L E D 2}$, $Z_{L E D 2}$. Adding them with the coefficients $k_{1}$ and $k_{2}$, one can get the resulting light with the chromaticity coordinates $X_{c}, Y_{c}, Z_{c}$ :

$X_{c}=k_{1} \cdot X_{L E D 1}+k_{2} \cdot X_{L E D 2}, Y_{c}=k_{1} \cdot Y_{L E D 1}+k_{2} \cdot Y_{L E D 2}$,

$Z_{c}=k_{1} \cdot Z_{L E D 1}+k_{2} \cdot Z_{L E D 2}$.

Since the $Y$ coordinate is brightness, when finding the coefficients $k_{1}$ and $k_{2}$, we can calculate the ratio of the LED brightnesses. One of three possible variants of determining these coefficients from the system of equations (8) have the form:

$k_{1}=\frac{Z_{c}-k_{2} \cdot Z_{L E D 2}}{Z_{L E D 1}}$,

$k_{2}=\frac{X_{c} \cdot Z_{L E D 1}-Z_{c} \cdot X_{L E D 1}}{X_{L E D 2} \cdot Z_{L E D 1}-Z_{L E D 2} \cdot X_{L E D 1}}$.

Thus, one can get the ratio of brightnesses for two white LEDs with various correlated color temperatures, when the correlated color temperature of their mixed white light will match a given color temperature in the Planckian locus.

Program implementation of the demonstrated model for mixing of colors of two white luminophore LEDs in SLS will provide the ability to change the correlated color temperature of white light in according to an arbitrarily given algorithm of change, including the natural cycle. But the question how to obtain different color light remains open. Solution of this question will allow to expand the functional possibilities of the lighting system and to increase the field of its application. This problem is solved through the use in SLS of RGBW LEDs, the mathematical model of color mixing for which will be discussed below.

\section{Mathematical model of color mixing of RGBW LEDs}

Now consider finding the ratio of the RGB LED brightnesses to obtain a given color temperature in SLS. To do this, it is necessary to measure their emission spectra at maximum brightness. It will provide a transition matrix with XYZ in the RGB space. Using this matrix, one can calculate the ratio of brightnesses between red, green and blue LEDs for resulting light with some brightness and correlated color temperature [8]. The transition matrix consists of the values $X_{R}, Y_{R}$, $Z_{R}$ for red LED, $X_{G}, Y_{G}, Z_{G}$ for green LED and $X_{B}, Y_{B}, Z_{B}$ for blue LED at their maximum brightness. If one knows the coordinates $x_{r}, y_{r}, x_{g}, y_{g}$ and $x_{b}, y_{b}$ for each color of RGB LEDs, from the formula (7) he can calculate their coordinates $X, Y, Z$ and plot a transition matrix from the RGB space into the XYZ space. This matrix has the following form:

$M=\left[\begin{array}{ccc}X_{R} & X_{G} & X_{B} \\ Y_{R} & Y_{G} & Y_{B} \\ Z_{R} & Z_{G} & Z_{B}\end{array}\right]$.

To find the value of correlated color temperature and light brightness, one can calculate the ratio of the brightnesses between emitting elements of RGB LED as follows:

$$
\left[\begin{array}{c}
R \\
G \\
B
\end{array}\right]=\left[\begin{array}{ccc}
X_{R} & X_{G} & X_{B} \\
Y_{R} & Y_{G} & Y_{B} \\
Z_{R} & Z_{G} & Z_{B}
\end{array}\right]^{-1} \cdot\left[\begin{array}{c}
X \\
Y \\
Z
\end{array}\right],
$$

where $R, G, B$ are the brightness of red, green and blue LEDs.

This system enables to obtain a large range of colors, the coordinates of which are limited by the Maxwell triangle (Fig. 3), but the resulting white light will have a color rendering index that is much lower (no more than 50) than that in WW LED system, for which this parameter is usually more than 75 . The increase in the color rendering index can be achieved by adding a range of white or orange LED. 
Let's consider the model of color mixing in SLS based on RGBW LEDs. Fig. 3 shows the Maxwell triangle, vertices of which are chromaticity coordinates of LEDs, from which RGB LED consists, and the point $W_{w}$ that are the coordinates of white LED. All the variants of colors with the values of chromaticity coordinates locating in the middle of the triangle are to be found using RGB or RGBW LEDs. It should be noted that usually, when mixing of emission of three components of LEDs in RGB or four components of LEDs in RGBW, at maximum brightness of each of them, one will get the points in the chromaticity diagram $W_{R G B}$ and $W_{R G B W}$ that are not located in the Planckian locus. The coordinates of the point $W_{R G B W}$ can be found as follows:

$$
\begin{aligned}
& X=X_{R}+X_{G}+X_{B}+X_{W}, \\
& Y=Y_{R}+Y_{G}+Y_{B}+Y_{W}, \\
& Z=Z_{R}+Z_{G}+Z_{B}+Z_{W},
\end{aligned}
$$

where $X_{W}, Y_{W}, Z_{W}$ - chromaticity coordinates of white luminophore LEDs.

Because chromaticity coordinates of obtained light do not localized in the Planckian locus, it is necessary to enter the coefficients $r, g, b, w$, to adjust the brightness of each LED so that the resulting chromaticity coordinates fulfilled it:

$$
\begin{aligned}
& \left\{\begin{array}{l}
X=r X_{R}+g X_{G}+b X_{B}+w X_{W}, \\
Y=r Y_{R}+g Y_{G}+b Y_{B}+w Y_{W}, \\
Z=r Z_{R}+g Z_{G}+b Z_{B}+w Z_{W}
\end{array}\right. \\
& \text { or }\left[\begin{array}{c}
X \\
Y \\
Z
\end{array}\right]=\left[\begin{array}{cccc}
X_{R} & X_{G} & X_{B} & X_{W} \\
Y_{R} & Y_{G} & Y_{B} & Y_{W} \\
Z_{R} & Z_{G} & Z_{B} & Z_{W}
\end{array}\right] \cdot\left[\begin{array}{c}
r \\
g \\
b \\
w
\end{array}\right] .
\end{aligned}
$$



Fig. 3. The Maxwell triangle for RGB LEDs.
Eq. (13) is convenient for calculating chromaticity coordinates of resulting radiation as it is done in other systems [9, 10], but to find four unknown $r, g, b, w$ coefficients in this system it requires a fourth equation. To do this, one should use the equation to get a certain point of the Maxwell triangle space with the known values $X Y Z$. This point can be $W_{w}$ that has chromaticity coordinates of white LED added into SLS. The situation, in which the color and brightness of white LED match those of light obtained by using red, green and blue LEDs, is described by the formula:

$r_{W} Y_{R}+g_{W} Y_{G}+b_{W} Y_{B}-Y_{W}=0$,

where $r_{W}, g_{W}, b_{W}-$ coefficient values of brightness of red, green and blue LEDs, which are calculated from Eq. (11) for obtaining light with color temperature and brightness, they match white LED $W_{w}$.

From the equation system (13), taking into account the expression (14) the coefficients $r, g, b, w$ are determined as follows:

$$
\left[\begin{array}{c}
r \\
g \\
b \\
w
\end{array}\right]=K_{R G B W}^{-1}\left[\begin{array}{c}
X \\
Y \\
Z \\
0
\end{array}\right]=\left[\begin{array}{cccc}
X_{R} & X_{G} & X_{B} & X_{W} \\
Y_{R} & Y_{G} & Y_{B} & Y_{W} \\
Z_{R} & Z_{G} & Z_{B} & Z_{W} \\
r_{W} Y_{R} & g_{W} Y_{G} & b_{W} Y_{B} & -Y_{W}
\end{array}\right]^{-1}\left[\begin{array}{c}
X \\
Y \\
Z \\
0
\end{array}\right]
$$

Setting the brightness of RGBW LEDs in proportion to the values of the corresponding coefficients, we obtain the resulting light with given chromaticity coordinates.

Software implementation of the demonstrated above model of color mixing of RGBW LED in SLS will provide an opportunity to obtain white light with different color temperatures with a high color rendering index. The luminous efficiency of lighting systems based on RGBW LED is less than that for the systems, in which two white LEDs are used. Such systems allow to obtain, first, white light with the coordinates in the Planckian locus, and, second, - light with various shades and colors.

\section{Experimental studies of the mathematical model of color mixing of WW and RGBW LEDs}

To test the mathematical models of mixing colors of two white luminophore LEDs with various correlated color temperatures and RGBW LEDs, the model of SLS was used. It is a lamp of "Armstrong" type with the size $60 \times 60 \mathrm{~cm}$ (Fig. 4), in which as an optical system used was a combination of two plastics - a transparent one with covered (on its reverse side) system of microlenses, acting as a lightguide, and opaque one, acting as a scatterer. Two models of SLS were produced. In the first model, along the perimeter there were the lines with WW LEDs that alternated between them, in the second model - lines with RGB and WW LEDs that are also alternating between them. 


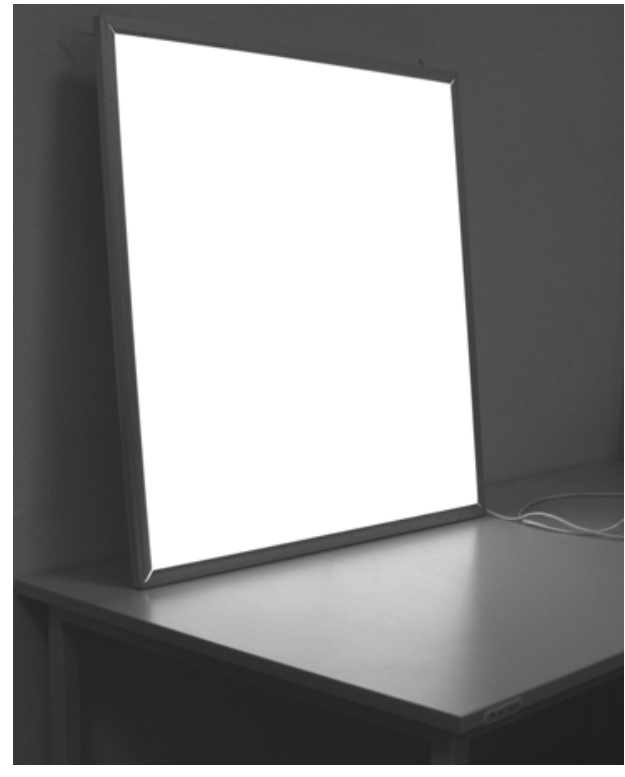

Fig. 4. Model of smart lighting system.

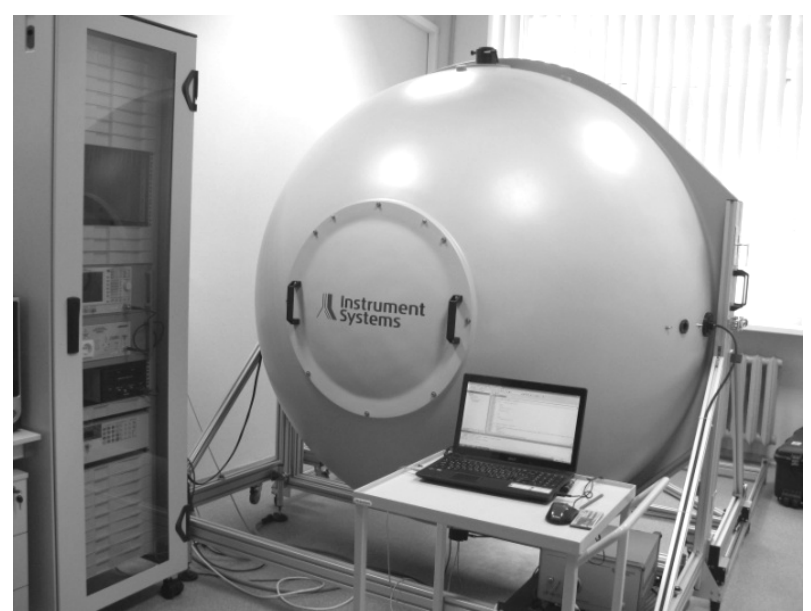

Fig. 5. Complex for measuring the light and electrical parameters of lighting systems.

To measure performance lighting engineering characteristics of SLS models, used was the measuring setup (Fig. 5), consisting of integrating sphere with the 2-m diameter, a spectrophotometer, power supplies and PC with the appropriate software. Using it, we measured spectra and $X Y Z$ coordinates of LEDs that were applied to create models of WW (Fig. 6) and RGBW (Fig. 7) smart lighting systems.

For WW LEDs, it was obtained $X_{L E D 1}=1150$, $Y_{L E D 1}=1025, Z_{L E D 1}=319$ and $X_{L E D 2}=1007, Y_{L E D 2}=$ $1100, Z_{L E D 2}=968$ with corresponding coordinates
$x_{L E D 1}=0.4610, y_{L E D 1}=0.4110$ and $x_{L E D 2}=0.3275$, $y_{L E D 2}=0.3577$.

The measured $X Y Z$ coordinates for RGB and WW LEDs allow to calculate and write the transition matrix based on Eq. (15):

$$
K_{R G B W}=\left[\begin{array}{cccc}
148.53 & 23.66 & 91.14 & 102.53 \\
62.13 & 97 & 17.21 & 100 \\
0.02 & 13.54 & 483.06 & 69.43 \\
31.36 & 66.50 & 2.14 & -100
\end{array}\right] .
$$

With these results, we have performed calculations for brightness of some emitting elements of SLS models to obtain white light with the given color temperature and have held its direct determination by using a setup for measuring lighting and electric parameters of lighting systems.

Listed in Table for two variants of SLS models are the given values of correlated color temperature $\left(T_{g}\right)$ and chromaticity coordinates $\left(x_{g}, y_{g}\right)$, experimentally obtained values of correlated color temperature $\left(T_{o}\right)$ and chromaticity coordinates $\left(x_{o}, y_{o}\right)$, as well as absolute values of the difference between them $\left(\Delta T=T_{o}-T_{g}\right.$, $\Delta x=x_{o}-x_{g}$ and $\left.\Delta y=y_{o}-y_{g}\right)$.


Fig. 6. The emission spectra of two white luminophore LEDs with correlated color temperature: a) $2700 \mathrm{~K}$, b) $5700 \mathrm{~K}$. 
Table. Results of studying the mathematical models of color mixing in WW and RGBW LEDs in smart lighting systems.

\begin{tabular}{|c|c|c|c|c|c|c|c|c|c|}
\hline \multirow{2}{*}{$\begin{array}{l}\text { Number of } \\
\text { measuring }\end{array}$} & \multicolumn{3}{|c|}{ Given values } & \multicolumn{3}{|c|}{ Obtained values } & \multirow{2}{*}{$\Delta T, K$} & \multirow{2}{*}{$\Delta x$} & \multirow{2}{*}{$\Delta y$} \\
\hline & $T_{g}, K$ & $x_{g}$ & $y_{g}$ & $T_{o}, K$ & $x_{o}$ & $y_{o}$ & & & \\
\hline \multicolumn{10}{|c|}{ For WW LEDs } \\
\hline 1 & 3200 & 0.4228 & 0.3958 & 3201 & 0.4217 & 0.3953 & 1 & -0.0011 & -0.0005 \\
\hline 2 & 3700 & 0.3946 & 0.3845 & 3700 & 0.3944 & 0.3844 & 0 & -0.0002 & -0.0001 \\
\hline 3 & 4200 & 0.3724 & 0.3756 & 4197 & 0.3732 & 0.3759 & -3 & 0.0008 & 0.0003 \\
\hline 4 & 4700 & 0.3546 & 0.3685 & 4687 & 0.3560 & 0.3692 & -13 & 0.0014 & 0.0007 \\
\hline 5 & 5200 & 0.3401 & 0.3627 & 5186 & 0.3410 & 0.3631 & -14 & 0.0009 & 0.0004 \\
\hline \multicolumn{10}{|c|}{ For RGBW LEDs } \\
\hline 6 & 3400 & 0.4110 & 0.3935 & 3411 & 0.4119 & 0.3970 & 11 & 0.0009 & 0.0035 \\
\hline 7 & 4400 & 0.3644 & 0.3661 & 4370 & 0.3646 & 0.3626 & -30 & 0.0002 & -0.0035 \\
\hline 8 & 5400 & 0.3348 & 0.3431 & 5410 & 0.3345 & 0.3416 & 10 & -0.0003 & -0.0015 \\
\hline 9 & 6100 & 0.3202 & 0.3300 & 6109 & 0.3200 & 0.3308 & 9 & -0.0002 & 0.0008 \\
\hline 10 & 7200 & 0.3038 & 0.3139 & 7217 & 0.3035 & 0.3143 & 17 & -0.0003 & 0.0004 \\
\hline
\end{tabular}
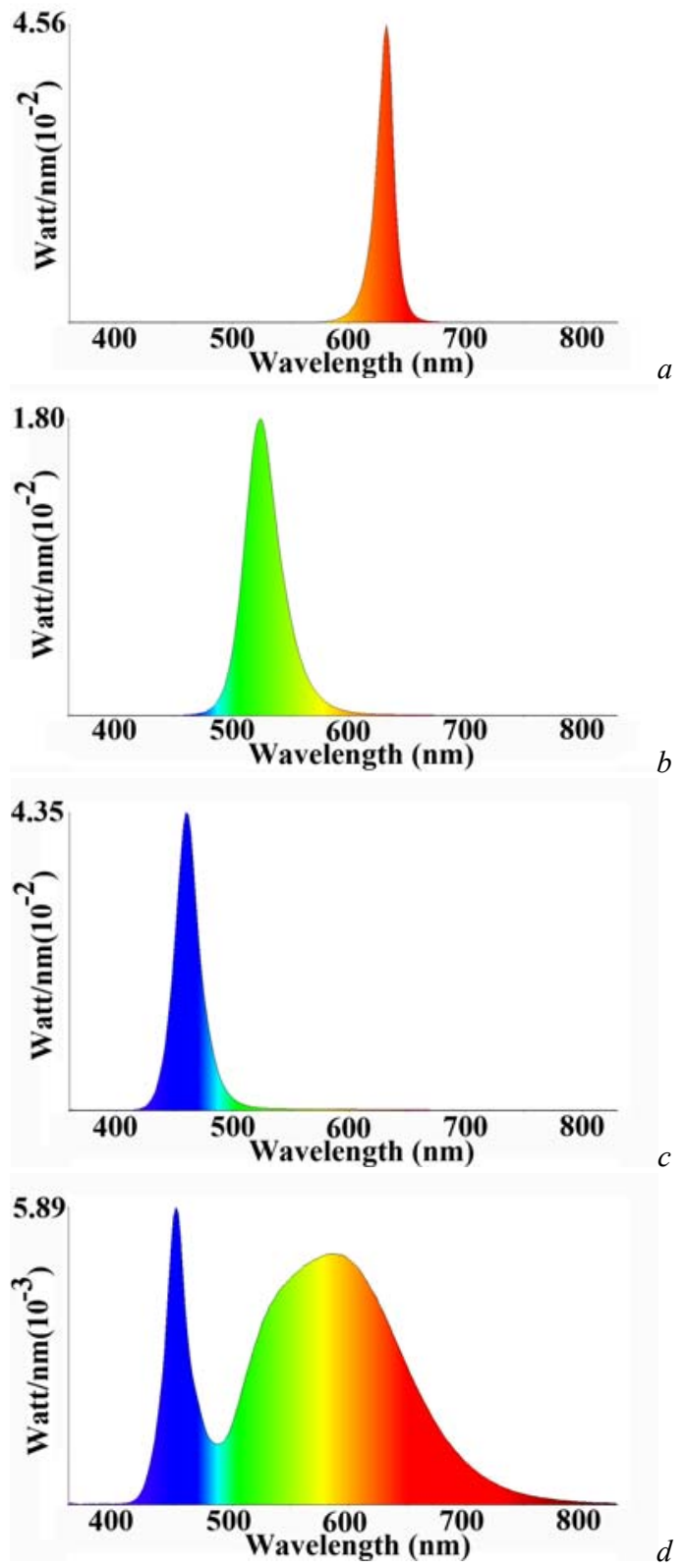

Fig. 7. The emission spectra: a) red (R), b) green $(\mathrm{G})$, c) blue (B) and d) white (W) LEDs.
The data in Table shows that the WW LED-based model demonstrated the possibility to obtain white light with the difference between the obtained and given values of chromaticity coordinates in the range 0.0014 and the values of color temperature - in $14 \mathrm{~K}$. This difference in chromaticity coordinates is well in the limits of experimental errors in these parameters measurements, which equals to \pm 0.0015 for the used complex. For such a value of measurement error in the chromaticity coordinates, the ranges of allowable values of correlated color temperature for their limit values are as follows: $(3159 \ldots 3240) \mathrm{K}$ for $3200 \mathrm{~K}$ and (7083...7278) $\mathrm{K}$ for $7200 \mathrm{~K}$.

For the RGBW LED-based model of SLS, it was determined the maximum differences between the obtained and given values in the chromaticity coordinates and in the correlated color temperature: 0.0035 and $30 \mathrm{~K}$, respectively. The revealed large difference in these parameters regarding the model of system on WW LEDs can be explained by the increasing number of LEDs of different types, which leads to increase in the number of given parameters, and to increase in influence of factors such as the accuracy of location of LEDs, the temperature of individual crystals in them, its dependence on operating mode, etc. Despite the fact that the difference between given and obtained chromaticity coordinates exceeds the measurement error for some values of color temperature, the difference in color does not exceed the value of two-step MacAdam ellipse. This is significantly less than the 7-step MacAdam ellipses, using which the range of color difference is set when determining one of eight nominal values of color temperature according to the standard of chromaticity C78.377A (Fig. 8).

The resulting color difference in two MacAdam ellipses can be considered quite acceptable, also because it corresponds to the maximum level of discreteness in color according to the standard NEMA SSL-3 Binning Standard in binning LEDs by their luminous flux value, forward voltage value and chromaticity. 




Fig. 8. The difference between the chromaticity coordinates of obtained and given light in SLS based on RGBW LEDs, which involves at least two steps of MacAdam ellipses.

Note that more opportunities of RGBW systems in terms of getting the white colors directly in the Planckian locus and colors of other shades from the Maxwell triangle lead to higher specifications in terms of practical implementation of SLS (LED locations and ensuring uniformity of color mixing) as well as to control and ensure stability of LED parameters depending on the power mode. Controlling the brightness of lighting for both types of systems is carried out by proportional change in the effective value of current through individual crystals of all LEDs that consist them

\section{Conclusion}

Considered in this work are the mathematical models of mixing colors in SLS based on WW and RGBW LEDs. They were software implemented and tested on experimental samples of the lamps of type "Armstrong" with the size $60 \times 60 \mathrm{~cm}$.

Software implementation of the model of mixing colors of two white luminophore LEDs showed the ability to dynamically change the correlated color temperature of light with the difference between the given and obtained values no more than $14 \mathrm{~K}$, which is in the limits of measurement errors.

Software implementation of the model of mixing colors of RGBW LEDs demonstrated the possibility of obtaining white light with various color temperature, chromaticity coordinates of which are directly in the Planck locus. Unlike the WW system, it allows to expand the range of permissible values of color temperatures of white light, which is limited only by the area of the Maxwell triangle for RGB LEDs, as well as the ability to get the color of any shade from this triangle. The difference between the given and obtained values of color temperature of white light is in the twostep MacAdam ellipse, which corresponds to the maximum requirement of the standard NEMA SSL-3 Binning Standard in the accuracy of selection in the color when binning white LEDs by manufacturers.

The performed studies show promising application of the considered models of mixing colors in SLS for reproduction of shades of white light by any given algorithm of changes, including the possibility of implementing changes in lighting in according with natural circadian rhythm or other algorithms of change in the color temperature and brightness.

\section{References}

1. G.C. Brainard, J.P. Hanifin, J.M. Greeson, B. Byrne, G. Glickman, E. Gerner, M.D. Rollag, Action spectrum for melatonin regulation in humans: Evidence for a novel circadian photoreceptor // J. Neurosci. No.21, p. 14-20 (2001).

2. Yu.B. Aizenberg, G.R. Shakhparuniants, About concept of prediction for lighting engineering development // Svetotekhnika, №5, p. 2-4 (2000), in Russian.

3. V.M. Sorokin, Yu.V. Kolomzarov, N.P. MaslakHudyma, M.A. Mynyaylo, Organic light-emitting structures - XXI century technologies // Tekhnologiia $i$ konstruirovanie $v$ elektronnoi apparature, №1, p. 3-9 (2009), in Russian.

4. V.I. Kornaga, A. Oleinik, V.M. Sorokin, O.D. Halynskyi, Creation and optimization of parameters of smart lighting systems // Svitlotekhnika and elektroenergetika. Kharkiv. 3-4 (35-36), p. 22-32 (2013), in Russian.

5. Patent USPTO (US Patents and Trademarks Office) US 12/623, 657, 23.11.2009. Ch.R. Simmers, Three-color RGB LED color mixing and control by variable frequency modulation // Patent US 8339058 B2. 2009.

6. A.V. Aladov, S.B. Biriuchynskyi, M.V. Dubina, A.L. Zakgeim, M.N. Mizerov, Controlled colordynamically operational lamp with full-color lightemitting diode // Svetotekhnika, No.2, p. 13-18 (2012), in Russian.

7. F.E. Schubert, Light-Emitting Diodes. Nauka, Moscow, 2008 (in Russian), p. 377-378.

8. V. Kornaga, V. Sorokin, A. Rybalochka, O. Oliinyk, Formation of color characteristics of smart lighting systems // Proc. V Intern. Sci.Techn. Conf. "Lighting and Electrical Power Engineering: History, Problems and Prospects", Ternopil, 2015, p. 33-34.

9. Chul Lee, Vishal Monga, Power-constrained rgbto-rgbw conversion for emissive displays // Intern. Conf. on Acoustic, Speech and Signal Processing (ICASSP), 2014, p. R.1214-1218.

10. M.E. Miller, M.J. Murdoch, RGB-to-RGBW conversion with current limiting for OLED displays // J. SID, 17(3), p. 195-202 (2009). 